ISSN: 2386-3919 - e-ISSN: 2386-3927

DOI: https://doi.org/10.14201/et2016342131148

\title{
VENTAJAS DEL TRATAMIENTO INCLUSIVO DE LA DIVERSIDAD: PERSPECTIVAS DE LOS PRINCIPALES AGENTES ENCARGADOS DE SU DESARROLLO
}

\section{Advantages of inclusive diversity treatment: perspective of the principal agents responsible for its development}

\author{
Raúl GonzÁlez Fernández*, M. ${ }^{a}$ del Castañar Medina DomíngueZ** \\ y María C. DOMínguez GARRIDO***** \\ Universidad Nacional de Educación a Distancia (UNED) \\ *Correo-e: raulgonzalez@edu.uned.es \\ * Correo-e: tatinamedina@yahoo.es \\ ***Correo-e: cdominguez@edu.uned.es
}

Recibido: 16/1/2016; Aceptado: 25/9/2016; Publicado: 30/11/2016 Ref. Bibl. RAÚl GONZÁlEZ FERNÁNDEZ, M. ${ }^{a}$ DEL CASTAÑAR MEDINA DOMÍNGUEZ y MARÍA C. DOMÍNGUEZ GARRIDO. Ventajas del tratamiento inclusivo de la diversidad: perspectivas de los principales agentes encargados de su desarrollo. Enseñanza \& Teaching, 34, 2-2016, 131-148.

RESUMEN: Estudios recientes destacan la actitud inicialmente favorable del profesorado hacia el tratamiento inclusivo de la diversidad, siendo los verdaderos artífices de su desarrollo, al mismo tiempo que resaltan las numerosas ventajas que la inclusión educativa tiene para todo el alumnado y para la propia institución educativa. Así, mediante una metodología mixta que combina la búsqueda y el análisis de datos cuantitativos y cualitativos, se ha realizado una investigación que pretende poner de manifiesto la percepción que los docentes de Educación Infantil, Primaria y Secundaria Obligatoria tienen sobre las ventajas y efectos positivos de la inclusión educativa, comprobando, al mismo tiempo, si existen diferencias significativas en función de la etapa educativa y de los años de experiencia docente. Los datos obtenidos mediante un cuestionario y una entrevista diseñados a tal efecto evidencian que 
132 RAÚl GONZÁLEZ FERNÁNDEZ, M. ${ }^{2}$ DEL CASTAÑAR MEDINA DOMÍNGUEZ Y MARÍA C. DOMÍNGUEZ GARRIDO VENTAJAS DEL TRATAMIENTO INCLUSIVO DE LA DIVERSIDAD:

PERSPECTIVAS DE LOS PRINCIPALES AGENTES ENCARGADOS DE SU DESARROLLO

el profesorado considera que el tratamiento inclusivo de la diversidad tiene múltiples ventajas: para los alumnos con necesidades, especialmente en el ámbito del desarrollo afectivo, emocional y social; para el alumnado sin necesidades, contribuyendo a la formación y óptimo desarrollo de sus valores éticos y morales; y, en último lugar, para el funcionamiento de la propia institución educativa, fundamentalmente en el campo de la renovación pedagógica y actualización docente. Si bien, se han encontrado diferencias significativas en esta percepción dependiendo de la etapa educativa del profesorado y, en menor medida, en función de los años de experiencia docente. actitud.

Palabras clave: ventajas; inclusión; diversidad; percepción de los docentes;

SUMMARY: Recent studies have suggested an initially favorable teacher attitude towards inclusive diversity treatment, with these teachers being the true authors of its development, while at the same time, the numerous advantages of educational inclusion are apparent for both students and the educational system itself. Therefore, using a mixed methodology that combines the search and analysis of quantitative and qualitative data, a study was conducted in order to determine the perception of Early Childhood, Primary and Secondary school teachers regarding the advantages and positive effects of educational inclusion, while at the same time verifying whether or not significant differences exist based on educational stage and years of teaching experience. The data obtained via a questionnaire and interview designed for these purposes reveals that teachers find inclusive diversity treatment to have multiple advantages: for students with special needs, particularly in the area of affective, emotional and social development; for students without special needs, contributing to the development and optimal formation of their ethical and moral values; and, finally, for the functioning of the educational institution itself, mainly in the area of pedagogical renewal and teacher updating. However, significant differences were revealed in this perception based on the educational stage taught by the teachers, and to a lesser degree, based on their years of teaching experience.

Key words: advantages; inclusion; diversity; teacher perception; attitude.

\section{INTRODUCCIÓN}

En las últimas décadas, los diversos sistemas educativos de los países de nuestro entorno han hecho un gran esfuerzo por promover políticas favorables al Tratamiento Educativo de la Diversidad (TED), entendido este término como "la respuesta educativa a toda clase de alumnos, buena parte de ellos con dificultades para el aprendizaje» (Rodríguez Tejada, 2009: 56). Es evidente que el concepto de diversidad da cabida a todas las personas, cada una con sus diversas necesidades educativas debidas a múltiples factores, hecho que demanda unas medidas de atención a la diversidad de calidad y ajustadas a cada caso en concreto (Palomares y López, 2013). 
Nuestro país no ha sido ajeno a este hecho, y así se pone de manifiesto en las diferentes leyes educativas promulgadas desde el año 1990. En este sentido, merece especial atención la Ley Orgánica 2/2006, de 3 de mayo, de Educación (LOE), puesto que en la misma se introduce por primera vez el término inclusión en detrimento del término integración: "La escolarización del alumnado que presenta necesidades educativas especiales se regirá por los principios de normalización e inclusión y asegurará su no discriminación» (Título II, art. 74.1).

En relación a este enfoque, Sola, López y Cáceres (2009) consideran que la inclusión es una superación del concepto de integración y, que por lo tanto, supone la integración total. En este sentido, Escarbajal (2010) indica que la inclusión hace referencia a una actitud positiva de respuesta a la diversidad del alumnado, adaptando los centros educativos a la misma: «El concepto ha ido evolucionado hasta entender que la inclusión se centra en cómo apoyar a todo el alumnado para ofrecerle un aprendizaje satisfactorio» (Ramírez y Muñoz, 2012: 199).

Estos hechos constatan, pues, un verdadero cambio en la atención al alumnado con necesidades educativas: "A partir de los años noventa se produce, en todo el mundo, un gran ímpetu para promover la inclusión» (Gento y González, 2010: 46), siendo considerada en los últimos Informes PISA como uno de los factores básicos de éxito de los sistemas educativos (OECD, 2010).

La atención a la diversidad en situaciones inclusivas es considerada en la actualidad como un importante elemento de renovación pedagógica, enmarcada en gran medida dentro del paradigma de calidad total (Gento y González, 2014). Es evidente que cuando la inclusión se realiza de modo conveniente y con los requisitos necesarios tiene una influencia global en la calidad de la institución educativa (Gento y González, 2010): "Sólo un sistema educativo de calidad, inclusivo, integrador y exigente, garantiza la igualdad de oportunidades y hace efectiva la posibilidad de que cada alumno o alumna desarrolle el máximo de sus posibilidades» (LOMCE, 2013, Preámbulo I).

Ahora bien, no es menos cierto que para poder desarrollar una educación inclusiva de calidad es necesario que los centros educativos se conviertan en parte dinámica y activa de todo el proceso, facilitando la implicación de todo el profesorado (Sánchez y García, 2013). Dicho de otro modo, «el éxito o fracaso de la puesta en práctica de cualquier política inclusiva depende en gran medida de la opinión favorable que mantengan al respecto los profesionales de la educación» (Avramidis y Norwich, 2004: 25).

En una línea similar, Alemany (2004) añade que el éxito de la puesta en marcha de cualquier política educativa depende de múltiples factores, siendo uno de los más relevantes el relacionado con las perspectivas de los profesionales de la educación, puesto que en último término son los artífices de su aplicación y desarrollo. A este respecto, Tárraga y Tarín (2013) afirman que «el profesorado tiene una enorme relevancia en todo el proceso de innovación que implica una escuela integradora, y más aún si nos queremos encaminar hacia un proyecto de escuela inclusiva..., pero difícilmente se podrá avanzar hacia este proceso de cambio si se 
134 RAÚl GONZÁLEZ FERNÁNDEZ, M. ${ }^{2}$ DEL CASTAÑAR MEDINA DOMÍNGUEZ Y MARíA C. DOMÍNGUEZ GARRIDO VENTAJAS DEL TRATAMIENTO INCLUSIVO DE LA DIVERSIDAD:

PERSPECTIVAS DE LOS PRINCIPALES AGENTES ENCARGADOS DE SU DESARROLLO

lleva a cabo de espaldas a los principales actores educativos, docentes y futuros docentes..." (pp. 39-40).

García-García et al. (2013) señalan que «cambiar la acción educativa supone la implicación del profesorado sensible a la problemática de sus aulas y confiado en que es posible modificarla» (p. 129). En este sentido, aun encontrando diferencias significativas en la actitud que el profesorado tiene hacia las prácticas educativas inclusivas en el aula dependiendo de factores como la etapa educativa o la experiencia docente (Escribano y Martínez, 2013; Ramírez y Muñoz, 2012; Rodríguez, Blázquez y Cubo, 2007; Verdugo y Rodríguez, 2012), todas las investigaciones recientes sobre esta temática ponen de relieve la actitud inicialmente favorable del profesorado hacia el tratamiento inclusivo de la diversidad (González Fernández, 2014; Palomares, Domingo y González, 2015; Rodríguez Tejada, 2009; Tiwari, Das y Sharma, 2015), apuntando en todos los casos las múltiples ventajas y efectos positivos que la inclusión educativa tiene para el desarrollo del alumnado.

\subsection{Ventajas del tratamiento inclusivo de la diversidad}

Todo indica, pues, que la inclusión educativa reduce la inequidad de los sistemas educativos, aumenta las posibilidades de aprendizaje de los diferentes grupos sociales más vulnerables y favorece el desarrollo de la cohesión social (Duk Momad, 2014).

En una línea similar, González-Gil y Martín-Pastor (2013) indican que los centros que han puesto en marcha modelos inclusivos han percibido beneficios no solo en los alumnos, sino también en toda la comunidad educativa en su conjunto, destacando el compromiso de formación adquirido por el profesorado. Así, desde la necesidad de favorecer el desarrollo de un verdadero modelo inclusivo del TED, Ledesma (2013) recalca que la atención a la diversidad de una persona conlleva también mejoras para el resto: aprovechando la riqueza que aporta la diversidad en los centros educativos se favorece el desarrollo de las inteligencias múltiples de todos los presentes, el aprendizaje de la convivencia y la regulación de conflictos.

Gento (2002) afirma que la inclusión educativa reporta ventajas para el desarrollo efectivo, el rendimiento académico, la aceptación social y el funcionamiento general del centro en el que se desarrolla.

Según todo ello y de manera más precisa, se puede afirmar que el tratamiento inclusivo de la diversidad presenta ventajas para el alumnado con necesidades educativas, para el alumnado sin necesidades y para el funcionamiento de la propia institución educativa (Gento y González, 2010):

- En el caso de los alumnos con necesidades, la inclusión educativa favorece su desarrollo afectivo, especialmente en el ámbito de la autoestima y autoconcepto, desarrollando, al mismo tiempo, actitudes más positivas hacia la escuela y mostrando un mayor dominio de sí mismos. Tiene igualmente efectos positivos sobre su rendimiento académico (mejorando sus resultados) 


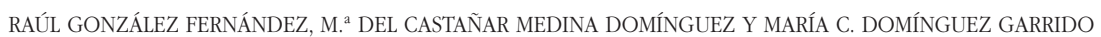

VENTAJAS DEL TRATAMIENTO INCLUSIVO DE LA DIVERSIDAD:

PERSPECTIVAS DE LOS PRINCIPALES AGENTES ENCARGADOS DE SU DESARROLLO

y proceso de adaptación social. Ahora bien, por encima de los resultados de tipo académico está el hecho de la aceptación social, viéndose ésta reforzada en el caso de los alumnos escolarizados en centros inclusivos.

- Para los alumnos sin necesidades, el tratamiento inclusivo de la diversidad parece ejercer una influencia positiva en el desarrollo de sus habilidades académicas básicas y en la mejora de sus relaciones sociales dentro y fuera del aula.

- En relación a la propia institución educativa, el desarrollo de la inclusión mejora el funcionamiento de la misma, dado que la inclusión educativa contribuye a dinamizar la actualización pedagógica del centro y de los docentes, siendo, por tanto, un factor importante de renovación pedagógica de centros y sistemas educativos.

No obstante, el tratamiento inclusivo de la diversidad reporta ventajas más allá de los propios alumnos y de la institución educativa, convirtiéndose en un elemento también importante en el desarrollo de una sociedad más justa, tolerante y respetuosa con la diversidad, sin olvidarnos de que la amplitud que tiene el término inclusión, enmarcado dentro del discurso de los derechos humanos, va mucho más lejos del ámbito educativo.

\section{OBjetivos}

En los párrafos anteriores se han puesto de manifiesto las ventajas que el tratamiento inclusivo de la diversidad ofrece, así como la importancia que la percepción y actitud del profesorado tiene como verdadero artífice de su desarrollo, en la aplicación de prácticas educativas inclusivas. Según estos argumentos, el objetivo principal de la presente investigación es clarificar la percepción que los docentes tienen sobre las ventajas que ofrece el tratamiento inclusivo de la diversidad.

Partiendo del mismo, y puesto que como ya se ha indicado en otros aspectos del TED parece haber diferencias en función de la etapa educativa y años de experiencia docente, esta investigación pretende también identificar las diferencias existentes en la percepción que el profesorado tiene sobre las ventajas de la inclusión en función de la etapa educativa en la que ejercen y en función de su experiencia docente.

\section{MÉTODO}

En esta investigación, predominantemente de tipo descriptivo, se ha utilizado una metodología mixta, que integra la búsqueda y análisis de datos cuantitativos y cualitativos, constituyendo, según Erickman y Roth (2006), dos fases de un proceso unitario a través de las cuales se puede obtener información relevante para el caso en cuestión. En este sentido, Ivankova (2015) señala que «el investigador 
136 RAúl GONZÁLEZ FERNÁNDEZ, M. ${ }^{a}$ DEL CASTAÑAR MEDINA DOMÍNGUEZ Y MARÍA C. DOMÍNGUEZ GARRIDO VENTAJAS DEL TRATAMIENTO INCLUSIVO DE LA DIVERSIDAD:

PERSPECTIVAS DE LOS PRINCIPALES AGENTES ENCARGADOS DE SU DESARROLLO

ha de tomar decisiones acerca del peso de los datos obtenidos en el contexto de la indagación y estimar durante las fases de la evaluación su relevancia, así como combinarlos o realizar la triangulación en el marco del estudio, al emplear métodos mezclados cuanti-cualitativos» (p. 206).

\subsection{Instrumentos}

Para la recogida de información, y tras el análisis de diversas fuentes documentales, se han diseñado de manera específica un cuestionario y una entrevista.

El cuestionario elaborado consta de diferentes partes: introducción, datos de identificación, 19 ítems de investigación y valoración del instrumento. Los ítems de investigación se agrupan en tres grandes bloques: percepción del profesorado sobre las ventajas que el tratamiento inclusivo de la diversidad tiene para el alumnado con necesidades educativas (mayor aceptación por parte de la sociedad, comunidad del centro, resto de compañeros, su propia familia y resto de familias; mejor desarrollo afectivo-emocional, intelectual, académico y social), para los estudiantes sin necesidades (mejor desarrollo social, afectivo-emocional e intelectual; mejora del comportamiento y aumento del rendimiento académico) y para el funcionamiento de la propia institución educativa (mayor profesionalización docente; mejoras en la organización y planificación de espacios; empleo de materiales didácticos más diversos; mejoras en la realización de la evaluación de todo el alumnado). Para responder a las diferentes cuestiones planteadas se ha optado por una escala tipo Likert de cinco grados, utilizando los siguientes cuantificadores de frecuencia: $1=$ nunca, $2=$ escasamente, $3=$ frecuentemente, $4=$ casi siempre y $5=$ siempre.

El instrumento inicialmente elaborado fue sometido a un juicio de expertos (tres profesores de la UNED y dos de la Universidad de Tubinga-Alemania-), y se realizó una aplicación inicial de carácter experimental a un total de ochenta y dos docentes, prestando especial atención al apartado de valoración del instrumento. Con toda la información recabada se elaboró el instrumento final, por lo que consideramos que se ha alcanzado una validez de contenido acorde con los requisitos de esta modalidad que va a permitir recoger los datos necesarios para la realización de la investigación planteada.

Del mismo modo, con el objetivo de determinar su fiabilidad y como paso previo al análisis de los datos obtenidos, se ha calculado el coeficiente Alfa de Crombach, alcanzando un índice de 0,895, por lo que se puede afirmar que el cuestionario aplicado evidencia una fiabilidad elevada.

La entrevista, de tipo semiestructurado, se ha preparado y organizado en función de los contenidos recogidos en el cuestionario, siguiendo sus dimensiones básicas, que aseguran la complementariedad de datos y su contraste en aquellas dimensiones esenciales constitutivas de ambas técnicas participativas.

Ambos instrumentos han permitido obtener datos relativos a la percepción que tienen los docentes sobre las ventajas del tratamiento inclusivo de la diversidad y, también, en función de las diferentes variables consideradas en la investigación: 
etapa educativa en la que el profesorado ejerce su docencia (Educación Infantil, Primaria o Secundaria Obligatoria) y años de experiencia docente del profesorado (entre 0-9; 10-20, o más de 20 años).

\subsection{Muestra}

La investigación realizada se ha llevado a cabo durante el curso 2014-15 en centros educativos públicos de todo el territorio nacional. En total se han recogido 2.518 cuestionarios, con presencia de todas las comunidades autónomas, siendo las más participativas Andalucía (16,7\%), Castilla La Mancha (12,1\%), Madrid (12,1\%) y Castilla y León (10,2\%). Por lo que se refiere a la etapa educativa (Figura 1), un 20,8\% del profesorado ejerce su labor en Educación Infantil, un 56\% en Educación Primaria y un 23,2\% en Educación Secundaria Obligatoria (ESO). Con respecto a los años de experiencia docente, un $54,8 \%$ se sitúa en el intervalo 0-9, un 29,2\% de los encuestados tiene una experiencia docente comprendida entre 10 y 20 años y un 16\% cuenta con más de 20 años.

FIGURA 1

Distribución de la muestra participante en la investigación

\section{Número de docentes por etapa educativa}

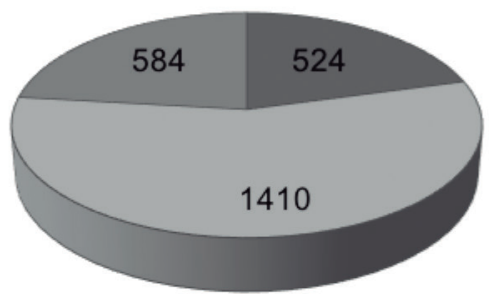

- Infantil aPrimaria $\square$ Secundaria

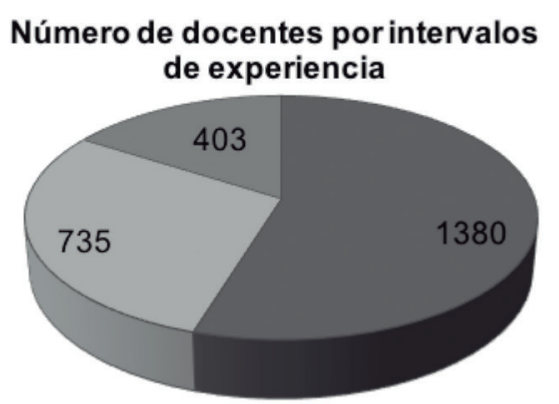

घ-9 años $\quad \square 10-20$ años $\square$ Más de 20

Igualmente, se han realizado 27 entrevistas a docentes que previamente habían cumplimentado el cuestionario, estando representadas todas las etapas educativas e intervalos de experiencia docente.

Morales Vallejo (2012) indica que a partir de una muestra de 2.401 sujetos existe un nivel de confianza del 95\%, admitiendo un margen de error del $2 \%$, por lo que podemos afirmar que la muestra final participante es suficientemente representativa y, por tanto, las descripciones y opiniones obtenidas son adecuadas al resto de docentes. 
138 RAÚl GONZÁLEZ FERNÁNDEZ, M. ${ }^{2}$ DEL CASTAÑAR MEDINA DOMÍNGUEZ Y MARÍA C. DOMÍNGUEZ GARRIDO VENTAJAS DEL TRATAMIENTO INCLUSIVO DE LA DIVERSIDAD:

PERSPECTIVAS DE LOS PRINCIPALES AGENTES ENCARGADOS DE SU DESARROLLO

\section{Resultados}

\subsection{Cuestionarios}

Los datos obtenidos a través de los cuestionarios han sido tratados con el programa estadístico sPss 22.0.

A nivel general, en relación a las ventajas que la inclusión educativa tiene para los estudiantes con necesidades educativas (Figura 2), el profesorado encuestado considera de manera bastante unánime que ésta contribuye fundamentalmente a su desarrollo afectivo y emocional $(\bar{X}=4,17)$, y así lo corroboran la desviación típica $(0,833)$ y los porcentajes obtenidos (siempre: 39,9\%; casi siempre: 41,6\%). Perciben también que la misma mejora su desarrollo social $(\bar{X}=4,14)$, favorece una mejor aceptación por parte de sus propios padres $(\bar{X}=4,11)$ y, en general, son mejor aceptados por parte de toda la comunidad en la que está ubicado el propio centro $(\bar{X}=4,01)$. Igualmente los encuestados consideran que, aunque en menor medida, el tratamiento inclusivo de la diversidad permite que los alumnos con necesidades sean mejor aceptados por el resto de sus compañeros $(\bar{X}=3,79)$, por la sociedad en general $(\bar{X}=3,75)$ y, del mismo modo, mejora su desarrollo intelectual $(\bar{X}=3,70)$. En menor medida, perciben que la inclusión tiene una ventaja inferior en lo que atañe a la mejora del rendimiento académico de los alumnos con necesidades $(\bar{X}=3,56$; escasamente: $15,1 \%$ ), y en lo relativo a la mejor aceptación por parte de las familias de sus compañeros sin necesidades $(\bar{X}=3,54$; escasamente: $12,9 \%)$.

FIGURA 2

Ventajas para estudiantes con necesidades educativas

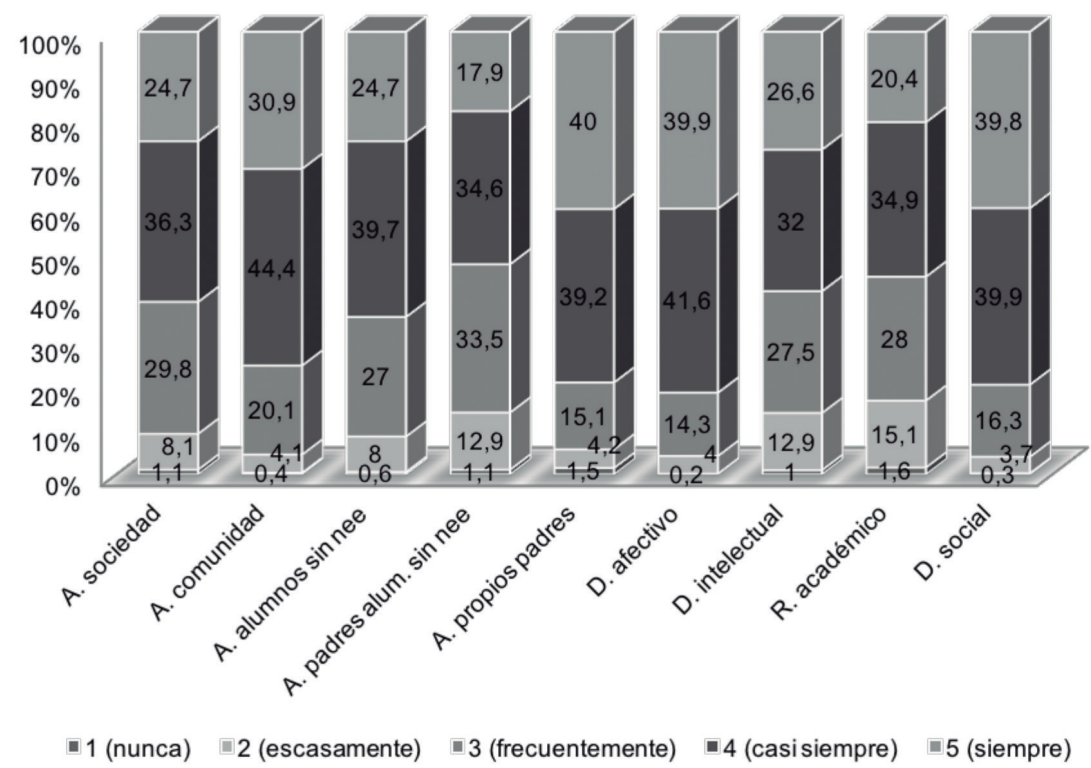


Por lo que se refiere a las ventajas que el tratamiento inclusivo de la diversidad tiene para el alumnado sin necesidades (Figura 3), los encuestados consideran que esta medida favorece un mejor desarrollo social de estos alumnos $(\bar{X}=4,09)$ y también contribuye a afianzar su desarrollo afectivo y emocional $(\bar{X}=4,07)$, y así se aprecia también en la desviación típica $(0,930$ y 0,935$)$ y porcentajes obtenidos (siempre: $40,1 \%$ y $38,8 \%$, respectivamente). Los encuestados perciben igualmente algunas ventajas en la mejora de su comportamiento $(\bar{X}=3,62)$ y desarrollo intelectual $(\bar{X}=3,10)$ y, en menor medida, consideran que favorece su rendimiento académico $(\bar{X}=2,92$; nunca: $8,8 \%)$.

FIGURA 3

Ventajas para estudiantes sin necesidades

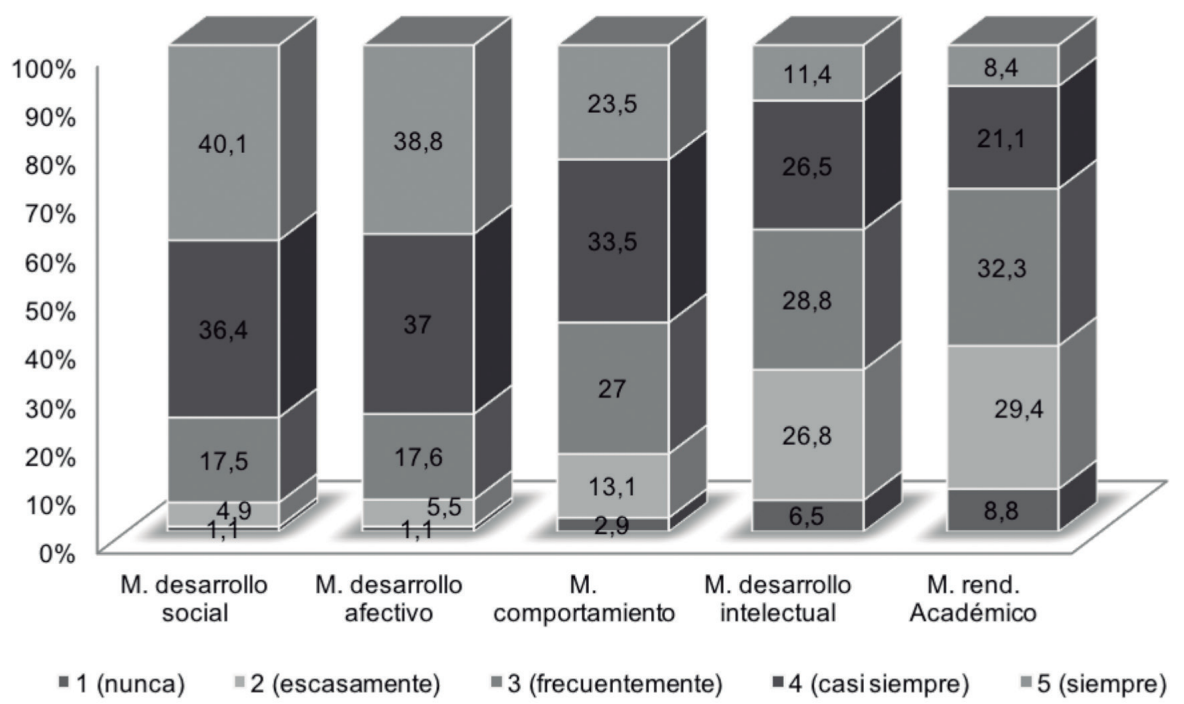

Finalmente, en relación a las ventajas que la inclusión tiene para el funcionamiento del propio centro educativo (Figura 4), el profesorado encuestado opina que ésta favorece la profesionalización de los docentes ( $\bar{X}=3,81$; siempre: $33,3 \%)$ y conlleva un mejor empleo de los diferentes materiales didácticos $(\bar{X}=3,70$; siempre: $26,3 \%)$. Tiene también ventajas para la organización del propio centro, planificación y utilización de los espacios $(\bar{X}=3,43$ y $\bar{X}=3,45)$. En menor medida, los encuestados perciben que la inclusión aporta algunas mejoras en el tema de la evaluación del alumnado ( $\bar{X}=3,35$; nunca: $5 \%$, escasamente: $18,4 \%)$. 
140 RAÚl GONZÁLEZ FERNÁNDEZ, M. ${ }^{2}$ DEL CASTAÑAR MEDINA DOMÍNGUEZ Y MARÍA C. DOMÍNGUEZ GARRIDO VENTAJAS DEL TRATAMIENTO INCLUSIVO DE LA DIVERSIDAD:

PERSPECTIVAS DE LOS PRINCIPALES AGENTES ENCARGADOS DE SU DESARROLLO

\section{FIGURA 4}

Ventajas para el funcionamiento del centro educativo

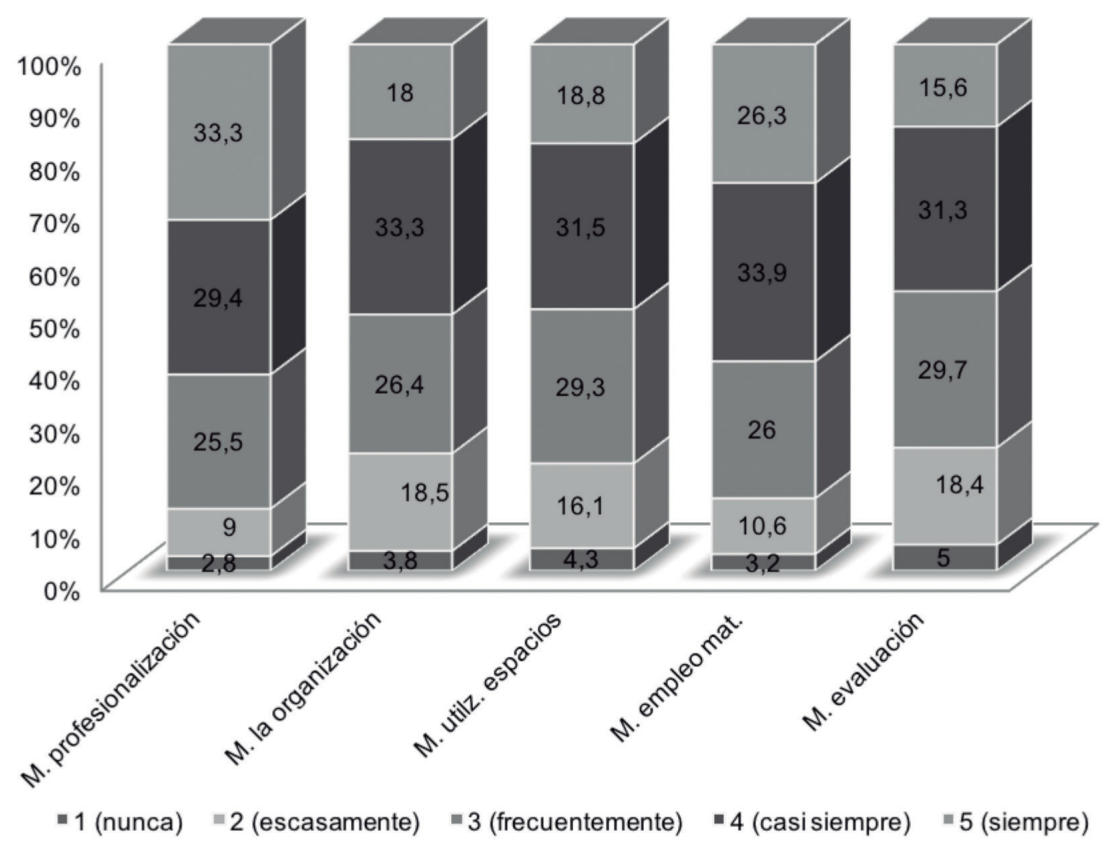

Por lo que se refiere a los resultados obtenidos en función de las diferentes variables consideradas, cabe señalar:

a) En relación a la etapa educativa en la que los docentes encuestados ejercen su actividad, en el caso de las ventajas para los estudiantes con necesidades educativas, se pueden apreciar, según la Prueba Ji-Cuadrado, diferencias estadísticamente significativas en todos los elementos considerados $(\mathrm{p}<, 05)$. En todos ellos se puede observar (Tabla 1) que, salvo en la aceptación por la sociedad, es el profesorado de Educación Infantil el que tiene una percepción superior de las ventajas que la inclusión educativa tiene para el alumnado con necesidades, seguido del profesorado de Educación Primaria. Por el contrario, a tenor de los datos obtenidos, todo apunta a que la percepción que el profesorado de la etapa de Educación Secundaria Obligatoria tiene sobre las posibles ventajas es manifiestamente inferior a la del resto de colegas. 


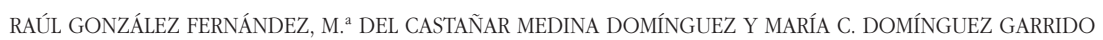

VENTAJAS DEL TRATAMIENTO INCLUSIVO DE LA DIVERSIDAD:

PERSPECTIVAS DE LOS PRINCIPALES AGENTES ENCARGADOS DE SU DESARROLLO

TABLA 1

Ventajas para estudiantes con necesidades educativas según la etapa educativa de los encuestados

\begin{tabular}{|c|c|c|c|c|c|c|c|c|c|}
\hline & 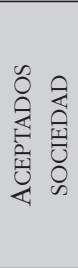 & 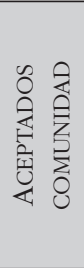 & 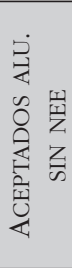 & 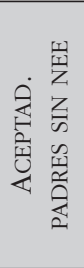 & 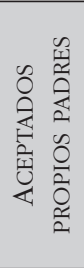 & 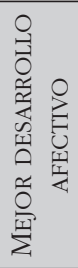 & 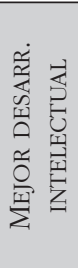 & 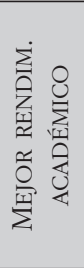 & 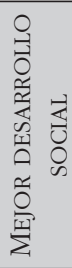 \\
\hline Ed. Infantil & 3,74 & 4,07 & 3,93 & 3,68 & 4,18 & 4,24 & 3,83 & 3,72 & 4,19 \\
\hline Ed. Primaria & 3,83 & 4,04 & 3,84 & 3,54 & 4,15 & 4,20 & 3,67 & 3,55 & 4,18 \\
\hline ESO & 3,58 & 3,89 & 3,58 & 3,47 & 4,01 & 4,04 & 3,66 & 3,49 & 4,02 \\
\hline
\end{tabular}

Algo similar sucede con las posibles ventajas para los estudiantes sin necesidades (Tabla 2). En este caso, tomando de nuevo como referencia la Prueba Ji-Cuadrado, existen también variaciones significativas en todos los elementos $(\mathrm{p}<, 05), \mathrm{y}$ todo indica que el profesorado de Secundaria es el que tiene una menor percepción de estas ventajas, siendo el profesorado de Educación Infantil el que percibe más ventajas en la inclusión del alumnado sin necesidades.

TABLA 2

Ventajas para estudiantes sin necesidades según la etapa educativa de los encuestados

\begin{tabular}{|l|c|c|c|c|c|}
\hline & $\begin{array}{c}\text { MEJOR DES. } \\
\text { SOCIAL }\end{array}$ & $\begin{array}{c}\text { MEJOR DES. } \\
\text { AFECTIVO }\end{array}$ & $\begin{array}{c}\text { MEJOR } \\
\text { COMPORT. }\end{array}$ & $\begin{array}{c}\text { MEJOR DES. } \\
\text { INTELECTUAL }\end{array}$ & $\begin{array}{c}\text { MEJOR RENDIM. } \\
\text { ACADÉMICO }\end{array}$ \\
\hline Ed. Infantil & 4,27 & 4,20 & 3,77 & 3,28 & 3,94 \\
\hline Ed. Primaria & 4,12 & 4,12 & 3,63 & 3,07 & 3,89 \\
\hline ESO & 3,85 & 3,84 & 3,46 & 2,99 & 3,50 \\
\hline
\end{tabular}

En el ámbito de las ventajas para el funcionamiento del propio centro (Tabla 3 ), se han encontrado igualmente diferencias significativas en todos los elementos $(\mathrm{p}<, 05) \mathrm{y}$, al igual que en los casos anteriores, las ventajas son menos perceptibles por el profesorado que ejerce su labor en la etapa de Educación Secundaria Obligatoria. Cabe señalar que, en el caso del empleo del material, el profesorado de educación primaria percibe ligeramente más ventajas $(\bar{X}=3,78)$ que sus colegas de infantil $(\bar{X}=3,75)$, teniendo una idea similar en lo que a mejora de evaluación se refiere $(\bar{X}=3,37)$. 
142 RAÚl GONZÁLEZ FERNÁNDEZ, M. ${ }^{2}$ DEL CASTAÑAR MEDINA DOMÍNGUEZ Y MARÍA C. DOMÍNGUEZ GARRIDO VENTAJAS DEL TRATAMIENTO INCLUSIVO DE LA DIVERSIDAD: PERSPECTIVAS DE LOS PRINCIPALES AGENTES ENCARGADOS DE SU DESARROLLO

TABLA 3

Ventajas para el funcionamiento del centro según la etapa educativa de los encuestados

\begin{tabular}{|l|c|c|c|c|c|}
\hline & $\begin{array}{c}\text { MEJOR } \\
\text { PROFESIONALIZACIÓN }\end{array}$ & $\begin{array}{c}\text { MEJOR } \\
\text { ORGANIZACIÓN }\end{array}$ & $\begin{array}{c}\text { MEJOR UTILIZAC. } \\
\text { ESPACIOS }\end{array}$ & $\begin{array}{c}\text { MEJOR EMPLEO } \\
\text { MATERIAL }\end{array}$ & $\begin{array}{c}\text { MEJOR } \\
\text { EVALUACIÓN }\end{array}$ \\
\hline Ed. Infantil & 3,94 & 3,56 & 3,53 & 3,75 & 3,37 \\
\hline Ed. Primaria & 3,89 & 3,47 & 3,48 & 3,78 & 3,37 \\
\hline ESO & 3,50 & 3,22 & 3,28 & 3,45 & 3,24 \\
\hline
\end{tabular}

b) Los resultados obtenidos en función de la variable años de experiencia docente evidencian menos diferencias estadísticamente significativas. En el caso de las ventajas para los estudiantes con necesidades educativas (Tabla 4), tan sólo se encuentran variaciones relevantes en el mejor desarrollo intelectual ( $X^{2}=30,294 ; \mathrm{p}$ $=, 000)$, el mejor desarrollo afectivo $\left(X^{2} \vee 26,543 ; \mathrm{p}=, 001\right)$, una mejor aceptación por parte de los alumnos sin necesidades $\left(X^{2}=21,177 ; \mathrm{p}=, 007\right)$, un mayor rendimiento académico $\left(X^{2}\right.$ v 17,$\left.897 ; \mathrm{p}=, 022\right)$ y un mejor desarrollo social $\left(X^{2}=16,104\right.$; $\mathrm{p}=, 041)$. Salvo en el caso del mejor desarrollo social, donde el profesorado con menos años de experiencia docente es el que percibe de manera más clara esta ventaja, todo parece apuntar a que el profesorado con mayor experiencia docente, seguidos por los que cuentan con menos años de docencia, ven de manera más clara las ventajas en el desarrollo intelectual, afectivo, académico y mayor aceptación por parte de sus compañeros.

TABLA 4

Ventajas para estudiantes con necesidades educativas según los años de experiencia de los encuestados

\begin{tabular}{|c|c|c|c|c|c|c|c|c|c|}
\hline & 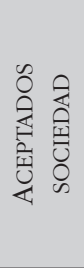 & 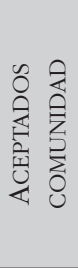 & 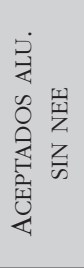 & 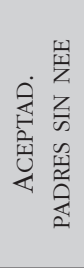 & 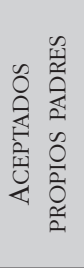 & 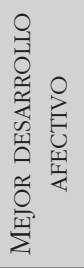 & 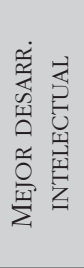 & 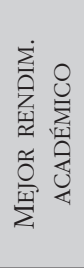 & 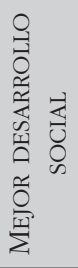 \\
\hline O-9 años & 3,76 & 4,01 & 3,76 & 3,54 & 4,12 & 4,18 & 3,69 & 3,57 & 4,18 \\
\hline O-20 años & 3,73 & 3,98 & 3,75 & 3,50 & 4,12 & 4,13 & 3,68 & 3,52 & 4,09 \\
\hline Más de 20 & 3,72 & 4,05 & 3,98 & 3,61 & 4,10 & 4,18 & 3,73 & 3,58 & 4,12 \\
\hline
\end{tabular}

En relación a las ventajas para los estudiantes sin necesidades (Tabla 5), se han encontrado diferencias representativas en el caso de la mejora del comportamiento $\left(X^{2}=22,582 ; \mathrm{p}=, 004\right)$, mejor rendimiento académico $\left(X^{2}=21,202 ; \mathrm{p}=, 007\right)$ y mejor desarrollo intelectual $\left(X^{2}=16,458 ; \mathrm{p}=, 036\right)$ : en estos casos es el profesorado con 


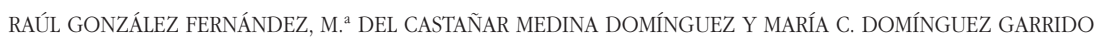

VENTAJAS DEL TRATAMIENTO INCLUSIVO DE LA DIVERSIDAD:

PERSPECTIVAS DE LOS PRINCIPALES AGENTES ENCARGADOS DE SU DESARROLLO

menor experiencia el que más percibe estas ventajas, seguido del profesorado con una experiencia docente comprendida entre 10 y 20 años.

TABLA 5

Ventajas para estudiantes sin necesidades según los años de experiencia de los encuestados

\begin{tabular}{|l|c|c|c|c|c|}
\hline & $\begin{array}{c}\text { MEJOR DES. } \\
\text { SOCIAL }\end{array}$ & $\begin{array}{c}\text { MEJOR DES. } \\
\text { AFECTIVO }\end{array}$ & $\begin{array}{c}\text { MEJOR } \\
\text { COMPORT. }\end{array}$ & $\begin{array}{c}\text { MEJOR DES. } \\
\text { INTELEC. }\end{array}$ & $\begin{array}{c}\text { MEJOR RENDIMI. } \\
\text { ACADÉMICO }\end{array}$ \\
\hline O-9 años & 4,12 & 4,09 & 3,68 & 3,15 & 2,95 \\
\hline 10-20 años & 4,06 & 4,03 & 3,55 & 3,02 & 2,84 \\
\hline Más de 20 & 4,02 & 4,02 & 3,49 & 2,98 & 2,82 \\
\hline
\end{tabular}

Finalmente, dentro de las ventajas para el funcionamiento del propio centro (Tabla 6), se han detectado variaciones significativas en todos los elementos $(\mathrm{p}<, 05)$. Salvo en el caso de la mejora en la utilización de los espacios, se puede afirmar que el profesorado con menor experiencia docente es el que tiene una mejor percepción sobre las múltiples ventajas que la inclusión ejerce en el funcionamiento de la institución educativa, siendo por el contrario el profesorado con más años de experiencia el que menos las percibe.

TABLA 6

Ventajas para el funcionamiento del centro según los años de experiencia de los encuestados

\begin{tabular}{|l|c|c|c|c|c|}
\hline & $\begin{array}{c}\text { MEJOR } \\
\text { PROFESIONALIZACIÓN }\end{array}$ & $\begin{array}{c}\text { MEJOR } \\
\text { ORGANIZACIÓN }\end{array}$ & $\begin{array}{c}\text { MEJOR UTILIZAC. } \\
\text { ESPACIOS }\end{array}$ & $\begin{array}{c}\text { MEJOR EMPLEO } \\
\text { MATERIAL }\end{array}$ & $\begin{array}{c}\text { MEJOR } \\
\text { EVALUACIÓN }\end{array}$ \\
\hline O-9 años & 3,87 & 3,46 & 3,45 & 3,76 & 3,38 \\
\hline 10-20 años & 3,74 & 3,43 & 3,41 & 3,61 & 3,30 \\
\hline Más de 20 & 3,69 & 3,33 & 3,46 & 3,59 & 3,22 \\
\hline
\end{tabular}

\subsection{Entrevistas}

Para el tratamiento de la información obtenida a través de las entrevistas efectuadas se ha utilizado el programa AQUAD 6, que posibilita el análisis cualitativo de textos.

A nivel general, se puede afirmar que los entrevistados opinan que el tratamiento inclusivo de la diversidad aporta ventajas a toda la comunidad educativa, impulsando el desarrollo de una sociedad más comprensiva, solidaria y tolerante. De manera más precisa, consideran que favorece el desarrollo integral de todo el alumnado, aportándoles conocimientos, aptitudes y actitudes que los habilitan de manera efectiva para el desarrollo adecuado de la vida en sociedad. 
144 RAÚl GONZÁLEZ FERNÁNDEZ, M. ${ }^{2}$ DEL CASTAÑAR MEDINA DOMÍNGUEZ Y MARíA C. DOMÍNGUEZ GARRIDO VENTAJAS DEL TRATAMIENTO INCLUSIVO DE LA DIVERSIDAD:

PERSPECTIVAS DE LOS PRINCIPALES AGENTES ENCARGADOS DE SU DESARROLLO

Concretamente, en el caso del alumnado con necesidades, los entrevistados creen que la inclusión favorece la evolución de todas sus capacidades y áreas en general: intelectual, afectiva, social, etc., desarrollando una actitud más positiva y estando más motivados hacia los temas académicos, mejorando, al mismo tiempo, su autoconcepto y autoestima, por lo que mejora la relación social con sus iguales y propias familias. No obstante, los encuestados subrayan fundamentalmente la igualdad de oportunidades: estudiar en un marco que reproduce la sociedad en la que se ubicarán en la vida adulta les prepara de manera efectiva para la vida futura. Del mismo modo, inciden en que el tratamiento inclusivo de la diversidad favorece la aceptación por parte de sus iguales y su integración en la comunidad en la que se ubica el centro y, por ende, en la sociedad en general, propiciando por tanto una verdadera integración social. Si bien, también es cierto que se han encontrado algunos matices particulares: el profesorado perteneciente a la etapa de Educación Primaria reduce las posibles ventajas en el caso de no contar con los recursos personales necesarios para la atención a las diferencias existentes en el aula. El profesorado de Educación Secundaria Obligatoria matiza todavía más las posibles ventajas, vinculándolas en mayor medida al desarrollo académico e indicando la necesidad de analizar la efectividad de la inclusión con el resto de sus compañeros.

Atendiendo a la variable años de experiencia, el profesorado con menos años de experiencia docente plantea el análisis de las ventajas en función del tipo de necesidad y grado de afectación, supeditándolas directamente a los recursos necesarios para su verdadera atención.

Por lo que se refiere a las ventajas para los estudiantes sin necesidades, los encuestados encuentran que la inclusión propicia su desarrollo intelectual, mejora su desarrollo afectivo y emocional y afecta positivamente a su comportamiento. Pero, sobre todo, inciden en que la inclusión favorece el desarrollo de valores éticos y morales (solidaridad, compromiso, respeto, colaboración, etc.) del alumnado sin necesidades, suponiendo además un aprendizaje para la vida que les va a permitir aceptar y respetar las diferencias y desarrollar sus habilidades sociales de comunicación e interrelación personal.

Se hace necesario recalcar que el profesorado que ejerce su labor educativa en la etapa de secundaria limita el impacto positivo de estas ventajas, haciendo más hincapié en la posible pérdida de tiempo de desarrollo de la materia que conlleva atender a la diversidad existente en el aula.

Según los entrevistados, el tratamiento inclusivo de la diversidad tiene también efectos positivos para el funcionamiento del propio centro. El desarrollo de la inclusión implica la realización de proyectos educativos más flexibles y abiertos a toda la comunidad, hecho que permite una mayor participación e implicación de las familias y resto de agentes sociales (servicios sociales y sanitarios, ayuntamiento, etc.), contribuyendo con ello a la promoción de la cultura de la igualdad y respeto a la diversidad.

Pero, al mismo tiempo, los entrevistados consideran que el tratamiento inclusivo de la diversidad contribuye en gran medida a la renovación de componentes 
RAÚL GONZÁLEZ FERNÁNDEZ, M. ${ }^{a}$ DEL CASTAÑAR MEDINA DOMÍNGUEZ Y MARÍA C. DOMÍNGUEZ GARRIDO

VENTAJAS DEL TRATAMIENTO INCLUSIVO DE LA DIVERSIDAD:

PERSPECTIVAS DE LOS PRINCIPALES AGENTES ENCARGADOS DE SU DESARROLLO

organizativos, funcionales y pedagógicos, es decir, invita a una actualización pedagógica que obliga a los docentes a organizar y planificar con más exactitud, buscar una mayor variedad de materiales didácticos y ampliar la propuesta de actividades y, por tanto, permite retomar y profundizar en el trabajo en equipo. Además, los encuestados, sobre todo los que tienen una mayor experiencia docente, recalcan que la inclusión educativa obliga al profesorado a una renovación formativa, a una formación continua en este ámbito y a un aumento de la profesionalización docente.

\section{DISCUSIÓN Y CONCLUSIONES}

Los resultados obtenidos evidencian que el profesorado de las etapas educativas de Educación Infantil, Primaria y Secundaria Obligatoria consideran que el tratamiento inclusivo de la diversidad reporta múltiples ventajas para los estudiantes con necesidades, sin necesidades y para el funcionamiento del propio centro, hechos que, a priori, repercuten en toda la comunidad educativa, contribuyendo al desarrollo de una sociedad más justa, tolerante y solidaria, al mismo tiempo que se facilita el desarrollo integral de la nuevas generaciones para su posterior integración efectiva en la vida en sociedad.

Si bien es cierto, estas ventajas suelen ser más visibles en el alumnado con necesidades, preparándole de manera efectiva para su vida futura, especialmente en lo que se refiere a una mejora en su desarrollo afectivo, emocional, social, etc., y, en consecuencia, aumentando su grado de aceptación por parte de su propia familia y de la comunidad educativa en la que se ubica el centro en cuestión. No obstante, la mejora en los aspectos anteriormente señalados también se considera que afecta en gran medida al alumnado sin necesidades, contribuyendo a su óptima formación y desarrollo de valores éticos y morales.

Parece que la inclusión educativa en el tratamiento educativo de la diversidad presenta menores ventajas en el campo de la mejora del rendimiento académico y escolar, especialmente en el caso de los alumnos con necesidades.

Pero estas ventajas van mucho más allá, incidiendo positivamente en el funcionamiento del centro en su conjunto, viéndose afectados por la misma todos los integrantes. Estas ventajas son muy evidentes en lo que atañe a la tan demandada renovación pedagógica, contribuyendo a una mejor profesionalización, formación y reciclaje continuo del profesorado -hecho que hace necesario que las administraciones educativas den respuesta en su justa medida-, incidiendo, en consecuencia, en la utilización por parte de los docentes de una metodología educativa más amplia y diversa, así como un mejor empleo de los diferentes materiales didácticos y recursos existentes. Dicho de otro modo, el tratamiento inclusivo de la diversidad conlleva abrir el centro a la sociedad, haciendo partícipe de la inclusión a toda la comunidad educativa y a los diferentes agentes sociales del entorno, desarrollando así el término inclusión en el sentido más amplio y poniendo en juego todos los elementos que son inherentes al mismo. 
146 RAúl GONZÁLEZ FERNÁNDEZ, M. ${ }^{a}$ DEL CASTAÑAR MEDINA DOMíNGUEZ Y MARÍA C. DOMÍNGUEZ GARRIDO VENTAJAS DEL TRATAMIENTO INCLUSIVO DE LA DIVERSIDAD:

PERSPECTIVAS DE LOS PRINCIPALES AGENTES ENCARGADOS DE SU DESARROLLO

Como ya se ha indicado anteriormente, investigaciones recientes han puesto de manifiesto la existencia de diferencias en determinados aspectos del tratamiento inclusivo de la diversidad, en función de la etapa educativa y de los años de experiencia docente del profesorado (Escribano y Martínez, 2013; González Fernández, 2014; Ramírez y Muñoz, 2012). Algo similar sucede con el tema de las posibles ventajas, según se indica a continuación.

El profesorado que ejerce su labor en la etapa de Educación Infantil, seguido del profesorado de la etapa de Primaria, percibe de manera más clara las diferentes ventajas que el tratamiento inclusivo de la diversidad tiene para todos los implicados. Por el contrario, es el profesorado de Educación Secundaria Obligatoria el que considera que la inclusión aporta ventajas de manera significativamente inferior al resto de sus colegas.

Por lo general, el profesorado de secundaria suele dar gran importancia a la evolución académica de sus alumnos, viendo más la labor docente desde la óptica de transmisores de conocimiento de una determinada materia. Por ello, no es de extrañar que los docentes de esta etapa hagan más hincapié en la relación existente entre ventajas-rendimiento académico, que como ya se ha indicado anteriormente es la ventaja menos perceptible por el profesorado, y la dedicación de tiempo que la atención a la diversidad supone.

Por lo que se refiere al tema de la experiencia docente, en términos generales, las diferencias existentes son menos evidentes y no siempre responden a un claro patrón. A modo de ejemplo, en el caso de los alumnos con necesidades parece que el profesorado con más años de experiencia, seguido por el profesorado con menos experiencia docente, son los que consideran que el tratamiento inclusivo de la diversidad reporta mayores ventajas para todos. No obstante, estos últimos vinculan su respuesta al grado de dificultad y a los recursos existentes en el centro, haciéndose verdadero por ello el miedo inicial hacia lo desconocido, donde es vital que el profesorado disponga de adecuada información y formación para dar una respuesta efectiva a la diversidad existente en el aula (Sánchez Palomino, 2011).

Dentro de las ventajas para el funcionamiento del propio centro, es el profesorado con menos años de experiencia el que más las percibe siendo, por el contrario, el profesorado con más años de carrera docente el más opuesto a esta afirmación, oposición que, en parte, se puede entender por el recelo que estos docentes suelen mostrar a la hora de introducir cambios en el ejercicio diario de su función docente y que, ineludiblemente, la inclusión educativa demanda.

Es por todo ello evidente que el profesorado de las etapas de Educación Infantil, Primaria y Secundaria Obligatoria percibe que el tratamiento inclusivo de la diversidad aporta múltiples ventajas a los estudiantes (con o sin necesidades), al propio centro educativo y, por extensión, a la sociedad en general. No obstante, se hace necesario seguir indagando en los posibles elementos y factores que inciden en una menor percepción de las mismas por parte del profesorado que ejerce su labor en la etapa de secundaria, analizando, al mismo tiempo, los factores que condicionan la variabilidad de respuestas en función de los años de experiencia 
RAÚl GONZÁLEZ FERNÁNDEZ, M. ${ }^{a}$ DEL CASTAÑAR MEDINA DOMÍNGUEZ Y MARÍA C. DOMÍNGUEZ GARRIDO

VENTAJAS DEL TRATAMIENTO INCLUSIVO DE LA DIVERSIDAD:

PERSPECTIVAS DE LOS PRINCIPALES AGENTES ENCARGADOS DE SU DESARROLLO

docente. Todo ello con el propósito de desarrollar el término inclusión en todas sus vertientes (escolar, social y comunitaria), dando así respuesta a la Estrategia 2020 de la Unión Europea, que tiene entre sus áreas prioritarias el fomentar el desarrollo de sociedades inclusivas, considerando este aspecto como un factor vital para el crecimiento.

\section{REFERENCIAS BIBLIOGRÁFICAS}

Alemany Arrebola, I. (2004). Las actitudes del profesorado ante el reto de integrar a alumnos con necesidades educativas especiales. Una propuesta de trabajo. Polibea, 72, 44-51.

Avramidis, E. y Norwich, B. (2004). Las actitudes del profesorado hacia la integración y la inclusión: revisión de la bibliografía de la materia. Entre Dos Mundos: Revista de Traducción sobre Discapacidad Visual, 25, 25-44.

Duk Momad, C. (2014). La formación y el desarrollo profesional de los docentes para una educación inclusiva. En A. Marchesi, R. Blanco y L. Hernández (Coords.). Avances y desafios de la educación inclusiva en Iberoamérica (pp. 61-70). Madrid: OEI.

Erickan, K. y Roth, W. M. (2006). What good is polarizing research into qualitative and quantitative? Educational Research, 35 (5), 14-23.

Escarbajal Frutos, A. (2010). La escuela inclusiva en una sociedad pluricultural y la importancia del trabajo colaborativo. Enseñanza \& Teaching, 28 (2), 161-179.

Escribano González, A. y Martínez Cano, A. (2013). Inclusión Educativa y Profesorado Inclusivo. Madrid: Narcea.

García-García, M.; Biencinto-López, C.; Carpintero-Molina, E.; Núñez-del-Río, M. C. y Arteaga-Martínez, B. (2013). Rendimiento en matemáticas y actitud hacia la materia en centros inclusivos: estudio en la Comunidad de Madrid. Revista de Investigación Educativa, 31 (1), 117-132.

Gento Palacios, S. (2003). Integración e inclusión educativa de calidad. En S. Gento Palacios (Coord.). Educación Especial I (pp. 195-288). Madrid: Sanz y Torres.

Gento Palacios, S. y González Fernández, R. (2010). Integración educativa e inclusión de calidad con personas con Necesidades Especiales Diversas. Madrid: UNED.

Gento Palacios, S. y González Fernández, R. (2014). Axiological Basis for a Curriculum Design in Educational Institutions of Quality: A View from Spain. En D. Brook Napier (Ed.). Qualities of Education in a Globalized World (pp. 131-148). The Netherlands: Sense Publishers.

González Fernández, R. (2014). Actitud del profesorado ante el Tratamiento Educativo de la Diversidad. Berlín: Publicia.

González-Gil, F. y Martín-Pastor, E. (2013). Formación del profesorado para la inclusión en España y Latinoamérica. En B. Vigo y J. Soriano (Coords.). Educación inclusiva: Desafíos y respuestas creativas (pp. 259-269). Zaragoza: Prensa Universitaria de Zaragoza.

Ivankova, N. V. (2015). Mixed Methods Applications in Action Research. California: SAGE Publications.

Ledesma Marín, N. (2013). Una escuela inclusiva cada vez más necesaria, también en tiempos de crisis. En M. J. Chisvert Tarazona, A. Ros Garrido y V. Horcas López (Coords.). A propósito de la inclusión educativa (pp. 17-26). Barcelona: Editorial Octaedro.

Ley Orgánica 2/2006, de 3 de mayo (BOE 4 de mayo), de Educación. 
148 RaÚl GONZÁLEZ FERNÁNDEZ, M. ${ }^{a}$ DEL CASTAÑAR MEDINA DOMÍNGUEZ Y MARÍA C. DOMÍNGUEZ GARRIDO VENTAJAS DEL TRATAMIENTO INCLUSIVO DE LA DIVERSIDAD: PERSPECTIVAS DE LOS PRINCIPALES AGENTES ENCARGADOS DE SU DESARROLLO

Ley Orgánica 8/2013, de 9 de diciembre ( $B O E 10$ de diciembre), para la Mejora de la Calidad Educativa.

Morales Vallejo, P. (2012). Tamaño necesario de la muestra: ¿Cuántos sujetos necesitamos? Descargado el día 18 de enero de 2015. http://web.upcomillas.es/personal/peter/investigacion/Tama\%F1oMuestra.pdf.

OECD (2010). PISA 2009 Results: Executive Summary. París: OECD.

Palomares Ruiz, A.; Domingo Gómez, B. y González Fernández, R. (2015). The educational intervention undertaken as regards students with communication difficulties enrolled in Pervasive Development Disorder (PDD) classrooms, in Bilingual and Preferential Attention Schools in the Community of Madrid (Spain). Journal of Education \& Social Policy, 2 (4), 80-84.

Palomares Ruiz, A. y López Sánchez, S. (2013). Los Programas de Cualificación Profesional Inicial y la atención a la diversidad en Castilla-La Mancha. Enseñanza \& Teaching, 31 (2), 23-44.

Ramírez García, A. y Muñoz Fernández, M. C. (2012). Prácticas inclusivas de los docentes en la convivencia escolar y en la organización y funcionamiento de los centros de Educación Primaria de la zona norte de Córdoba. Revista de Investigación Educativa, 30 (1), 197-222.

Rodríguez Tejada, R. M. (2009). La Atención a la Diversidad en la ESO. Actitudes del Profesorado y Necesidades Educativas Especiales. Badajoz: Diputación de Badajoz.

Rodríguez Tejada, R. M.; Blázquez Entonado, F. y Cubo Delgado, S. (2007). Profesores de ESO y necesidades educativas especiales. Enseñanza E Teaching, 25, 111-134.

Sánchez Palomino, A. (2011). La Universidad de Almería ante la integración educativa y social de los estudiantes con discapacidad: ideas y actitudes del personal docente e investigador. Revista de Educación, 354, 575-603.

Sánchez Sainz, M. y García Medina, R. (2013). Diversidad e inclusión educativa. Madrid: Catarata.

Sola Martínez, T.; López Urquízar, N. y Cáceres Reche, M. P. (2009). La educación especial en su enmarque didáctico y organizativo. Granada: Grupo Editorial Universitario.

Tárraga Mínguez, R. y Tarín Ibáñez, J. (2013). Escuela inclusiva: controversias en torno a discursos, políticas y actitudes. En M. J. Chisvert Tarazona, A. Ros Garrido y V. Horcas López (Coords.). A propósito de la inclusión educativa (pp. 27-53). Barcelona: Editorial Octaedro.

Tiwari, A.; Das, A. y Sharma, M. (2015). Inclusive education a "rheoric» or "reallity»? Teachers' perspectives and beliefs. Teaching and Teacher Education, 52, 128-136. 